\title{
Évaluation de \\ la capacité de réfrigération d'un estuaire Exemple de la Loire
}

\author{
par A. Daubert \\ Chef du Laboratoire National d'Hydraulique, \\ E.D.F., Chatou
}

\author{
ef J.-F. Malherbe \\ Ingénieur à la Division Recherches \\ Laboratoire National d'Hydraulique, E.D.F., Chatou
}

\section{Introduction}

Les eaux d'un estuaire subissent un mouvement de vaet-vient sous l'effet des marées et sont renouvelées plus ou moins rapidement suivant :

- le débit du fleuve;

- le taux d'échange de l'estuaire avec la mer, caractérisé par la partie du volume évacué au jusant qui est réintroduite aut flot.

Une même tranche d'eau, comprise entre deux sections droites, passera donc devant une centrale établie en un point donné de l'estuaire un certain nombre de fois, ce nombre étant déterminé par les paramètres convectifs que l'on vient de citer. A chaque fois, la tranche reçoit des calories qui se diluent sur toute sa largeur en plus ou moins de temps. Le temps d'une marée séparant deux passages consécutifs est, en général, suffisant pour que la répartition des calories soit complète et ceci sans que le refroidissement atmosphérique ait pu réduire leur nombre en proportion significative. Les tranches d'eau passant devant la centrale subiront donc des échauffements cumulatifs, ce qui est particulièrement gênant pour la température de prise. On notera que le cumul est quand même limité par le refroidissement atmosphérique, car le temps du cumul est relativement long et le refroidissement luimême se fait plus efficace au fur et à mesure que les températures augmentent.

L'échauffement dans la masse de l'estuaire est donc cause d'une recirculation que l'on peut à la fois qualifier de :

- partielle, en raison de la répartition des calories sur toute la largeur de l'estuaire; la recirculation est alors limitée par le domaine d'aspiration de la prise qui n'intéresse pas la totalité de cette largeur;

- différée, en raison du parcours effectué par les calo- ries; les températures sont alors atténuées par le refroidissement atmosphérique et uniformisées longitudinalement par des échanges dispersifs entre les tranches d'eau de l'estuaire.

La recirculation prend un visage différent suivant l'instant de la marée et la disposition relative du rejet et de la prise. En général, celle-ci est implantée à l'amont de celui-là. Au moment du flot, le transit entre rejet et prise sera court et l'on aura donc une recirculation directe au lieu de différée. Elle sera de plus totale, si la prise est située sur la même berge que le rejet, et assez proche pour que les calories n'aient pu être diluées latéralement. L'élévation de température, qui résulte à la prise de cette mauvaise répartition, doit alors être ajoutée à l'élévation fictive qu'on pourrait calculer en supposant une dilution immédiate du rejet.

On présente ici un modèle permettant de calculer les températures moyennes dans les sections droites de l'estuaire et, en particulier, dans celle de la prise. S'attacher aux valeurs moyennes conduit à adopter un point de vue unidimensionnel. Le modèle auquel on aboutit dans ces conditions n'est, en contrepartie, pas représentatif de la température maximum obtenue à la prise au moment du flot. Ce qui a été dit précédemment montre, en effet, que le problème est alors bidimensionnel. Mais il ne s'agit là que d'un problème local sans conséquence sur le régime thermique de l'estuaire. Les écoulement en estuaire ont également un aspect bidimensionnel assez marqué en raison de circulations transversales importantes (et un aspect tridimensionnel au moment des renverses, même pour les estuaires bien mélangés). L'effet des mouvements de convection réels sur le transport des effluents se présente comme une dispersion autour du transport déterminé par la convection moyenne (dans le sens longitudinal). Il a été démontré que cette dispersion pouvait être prise en compte par un terme de diffusion dans une description 
unidimensionnelle, et ceci rend l'obstacle non rédhibitoire. La dispersion accélère les transferts par rapport à la convection unidimensionnelle. Elle influe, par conséquent, sur la puissance déversée en mer, ce qui entraîne, corrélativement, une diminution du poste de refroidissement qu'est l'atmosphère. Ceci est favorable quant aux températures régnant dans l'estuaire puisque les échanges atmosphériques sont au premier ordre proportionnels aux échauffements.

\section{Le modèle d'estuaire}

Il adopte le point de vue filaire qui a été défini et rend compte des moyennes des différentes grandeurs dans les sections droites. Il comporte deux parties, l'une hydraulique, l'autre thermique.

\section{Partie hydraulique du modèle}

$$
\begin{aligned}
& \text { Elle est basée sur les équations de Saint-Venant : } \\
& \begin{array}{l}
L \frac{\partial z}{\partial t}+\frac{\partial Q}{\partial x}=q \quad \quad \text { (équation de continuité) } \\
\frac{\partial V}{\partial t}+\frac{\partial}{\partial x}\left(\frac{V^{2}}{2}+g z\right)=-g \frac{V|V| P^{4 / 3}}{K^{2} S^{4 / 3}} \\
\text { (équation de la dynamique) }
\end{array}
\end{aligned}
$$

où les notations suivantes représent :
$t \quad$ le temps;
$x \quad$ l'abcisse le long de l'axe de l'estuaire;
$z \quad$ la cote de la surface libre dans une section;
$L$ la largeur au miroir : c'est une fonction bi-uni- voque de $z$;
$Q \quad$ le débit;
$q \quad$ les sources ou puits de débit injecté ou prélevé par unité de longueur dans l'estuaire (en particulier à la prise);
$S \quad$ la section mouillée est fonction bi-univoque de $z$;
$V \quad$ la vitesse moyenne dans la section, autrement dit la vitesse débitante. Elle est égale à $Q / S$;
$P \quad$ le périmètre mouillé est fonction bi-univoque de $z$;
$K \quad$ un coefficient de Strickler variable avec $x$ et $z$.

Une fois la géométrie donnée, c'est-à-dire les relations entre $z, L, S$ et $P$, le modèle demande la définition des fonctions $q$ et $K$. Cette dernière est, en général, obtenue par étalonnage. Le système comporte donc, en fait, deux inconnues en fonction de $x$ et $t$, à choisir parmi les fonctions dynamiques $Q$ et $V$ d'une part et les fonctions géométriques $z, S, L$ et $P$ d'aurte part.

Afin de représenter le stockage et le temps de rétention qu'un canal de fuite éventuel peut introduire dans le système, le modèle prend en compte l'existence de trois bras :

- le premier est constitué par la partie de l'estuaire située à l'amont de la centrale;

- le second par le canal de fuite;

- le troisième par la partie de l'estuaire située à l'aval de la centrale.

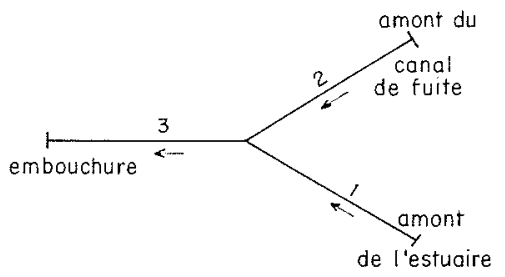

La jonction entre ces trois bras est assurée par le volume de confluent $\Omega$ occupé dans le lit de l'estuaire au droit de la prise, et mélangé par le débit des trois bras. Son évolution est représentée par :

$$
\begin{gathered}
z_{\omega}=z_{1}=z_{2}=z_{3} \\
\partial \Omega / \partial t)+Q_{1}+Q_{2}-Q_{3}=0
\end{gathered}
$$

Les indices corerspondent à chacun des trois bras. Le signe moins dans la seconde équation provient de l'orientation identique qui leur est donnée, de l'amont vers l'embouchure. $\Omega$ est une fonction bi-univoque de $z \omega$ qui doit être donnée.

Pour achever la définition du système, il faut lui adjoindre l'ensemble de ses conditions aux limites. Cet ensemble est ici constitué par une condition en chacune des extrémités des bras autres que le confluent (où une condition est déjà imposée). Ceci tient à la nature fluviale des écoulements dans les estuaires. Dans la pratique, les données que l'on peut se procurer le plus facilement sont aussi les paramètres essentiels du problème posé. Elles amènent à définir pour le système :

- le débit à l'amont du bras 1 (partie amont de l'estuaire); représenter ce bras jusqu'à la limite fluviale de l'estuaire permet d'imposer directement le débit du fleuve;

- le débit à l'amont du bras 2 (canal de fuite) : c'est le débit prélevé par la centrale;

- la cote à l'aval du bras 3 (partie aval de l'estuaire) : c'est elle qui traduira l'intensité de la marée.

On peut cependant modifier si besoin le type de ces lois et remplacer une loi de débit par une loi de cote ou vice-versa. En particulier, cette latitude s'avère utile si l'on ne veut pas représenter l'estuaire jusqu'à sa limite fluviale. On utilisera alors, à l'amont du bras 1, une loi de cote.

Dans certains cas, il pourra s'établir un régime critique à l'extrémité du canal de fuite. Il faudra alors retirer $z_{2}$ de la série d'égalités qui définissent les cotes au confluent et remplacer cette condition aval du bras 2 par la condition de criticité.

En fin de compte, la partie mécanique du modèle est apte à reproduire tous les mouvements de convection des tranches d'eau le long de l'estuaire. Ils interviennent de façon primordiale pour régler l'équilibre thermique de celui-ci en rendant compte :

- de l'évolution des débits au droit de la centrale;

- du stockage dans le canal de fuite;

- des distances de parcours au flot et au jusant.

Tous ces facteurs dépendent du débit du fleuve et de l'intensité de la marée. 


\section{Partie thermique du modèle}

Elle est basée sur l'équation de la conservation de la chaleur :

$$
\frac{\partial S T}{\partial t}+\frac{\partial Q T}{\partial x}+\frac{A}{C_{p}} L(T-T e q)=\frac{\partial}{\partial x}\left(S D_{x} \frac{\partial T}{\partial x}\right)
$$

où les notations suivantes représentent :

$T$ la température moyenne dans une section droite;

$C p \quad$ la chaleur volumique de l'eau à pression constante;

$A$ un coefficient d'échange atmosphérique linéarisé;

Teq la température de linéarisation;

$D \quad$ le coefficient de dispersion.

Cette équation représente les trois modes de transfer suivants :

\section{Les transferts convectifs}

Ils sont représentés par les deux premiers termes. La partie mécanique du modèle procure les éléments convectifs $Q$ et $S$.

\section{Les échanges atmosphériques}

Ils sont d'autant plus importants que la température s'écarte de la valeur Teq pour laquelle ils sont nuls. Cette valeur est déterminée conformément aux résultats présentés dans les travaux cités en référence [2]. Il y est également montré comment on peut faire une linéarisation autour de Teq par un coefficient d'échange de la forme

$$
A=a+b \sqrt{u^{2}+V^{2}}
$$

- $a$ est une constante résumant l'effet :

- des rayonnements (solaire, atmosphérique et iu plan d'eau),

- de la diffusion moléculaire (entre le ) en l'absence plan d'eau et l'air), de vent;

- de l'évaporation,

- $b$ est une constante résumant l'influence de la convection entre l'air et l'eau sur la diffusion moléculaire et sur l'évaporation;

- $u$ est la vitesse du vent; c'est en réalité la vitesse relative à celle de l'eau qui importe; la racine carrée, dans laquelle $u$ est inclus, permet de rendre compte de la moyenne statistique de cette vitesse relative, étant donné les changements de direction du vent.

$T e q, a, b$ et $u$ sont des paramètres climatologiques régionaux et restent constants à l'échelon d'une saison. Mais le coefficient d'échange $A$ est fonction de $x$ et $t$ par l'intermédiaire de $V$. On peut donc, si on le désire, améliorer la description des échanges atmosphériques en adoptant des lois de variation plus fines des paramètres en fonction de $t$, et aussi de $x$ si l'estuaire a une très large étendue. Cependant, plus on descend dans les échelles des temps et des longueurs, plus la nature aléatoire des échanges atmosphériques prend de l'importance.

\section{La dispersion}

Elle est causée par la disparité des vitesses dans une section droite. Il en résulte un mélange dans les volumes soumis aux mouvements convectifs longitudinaux moyens. L'analyse dimensionnelle conduit à poser

$$
D_{x}=\alpha V d
$$

où :

$\alpha \quad$ est un coefficient adimensionnel;

$V \quad$ une vitesse;

d une longueur.

A profil de vitesse donné dans les sections droites, les volumes des tranches intéressées par le mélange sont proportionnels au débit total. On est donc conduit à prendre un coefficient de la forme :

$$
D_{x}=\alpha(Q / d)
$$

Par ailleurs Fischer [6] a montré que ce sont surtout les mouvements de circulation latéraux qui donnent les écarts les plus importants entre la vitesse débitante et les vitesses réelles. Ceci incite à choisir, comme longueur de référence $d$, la largeur $L$ des sections droites. Les calculs effectués par D.W. Prichard [3] pour l'estuaire de l'Hudson confirme le bien-fondé de ce choix. Ainsi, $\alpha$ se présente comme un coefficient de forme lié au profil des débits par unité de largeur (les débits étant considérés dans cette représentation de $D_{v}$ comme uniformément répartis sur la profondeur). On a adopté en fait un coefficient de la forme :

$$
D_{x}=\alpha(Q / L)+\beta
$$

pour marquer que la dispersion n'est pas nulle même lorsque la vitesse débitante l'est. $\beta$, coefficient dimensionnel, est évidemment très faible devant le premier terme.

\section{Les conditions aux limites}

L'équation de la chaleur régit l'évolution des calories à l'intérieur de chacun des bras définis dans la partie hydraulique du modèle. Afin de bien poser le problème, il est nécessaire d'introduire une condition aux limites à l'extrémité de chaque bras. Ce peut être une température, un gradient ou une condition mixte.

La nature du problème conduit à imposer les conditions suivantes :

CONDITION A L'EXTRÉmité AMONT dU bRAS 1 (partie amont de l'estuaire) :

Puisque le bras s'étend jusqu'à la limite maritime de l'estuaire, la température à l'amont du bras est la température naturelle du fleuve $T_{F}$ qu'il est donc nécessaire de connaître.

Condition a l'extrémité amont du bras 2 (canal de fuite) :

Si $T_{p}$ est la température calculée dans le bras 1 au niveau de la prise et $Q_{P}$ le débit de prise, la puissance $P_{2}$ rejetée 
par la centrale se traduira par une condition de température :

$$
T_{2}(t)=T_{P}(t-\Delta t)+\Delta T_{P}
$$

avec :

$$
\Delta T_{P}=P_{2} / C_{P} Q_{P}
$$

( $\Delta t$ représente le temps de rétention de la centrale si on veut le prendre en compte.)

CONDITION A L'EXTRÉmité DU BRAS 3 (partie aval de l'estuaire) :

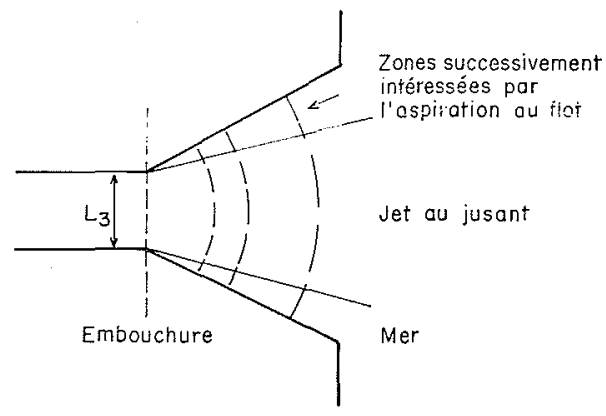

Au jusant, les eaux sortent de l'estuaire et il n'y a pas d'échanges avec la mer : $\left(\partial T_{3} / \partial t\right)+V_{3}\left(\partial T_{3} / \partial x\right)=0$.

$\mathrm{Au}$ flot, une partie des eaux sorties au jusant peuvent revenir dans l'estuaire, surtout les dernières. Mais elles auront subi une certaine dispersion en mer et reviendront moins chaudes. D'après des résultats théoriques et expérimentaux, présentés par Schlichting [7], on peut assimiler grossièrement la courbe de température moyenne d'un jet chaud diffusant en milieu calme à une droite. Cette droite décroît depuis la température $T_{3}$ à la sortie du jet (ici l'embouchure) jusqu'à la température du milieu ambiant, et ceci sur une longueur égale à 4 fois la largeur du jet (ici 4 fois la largeur $L_{3}$ de l'embouchure).

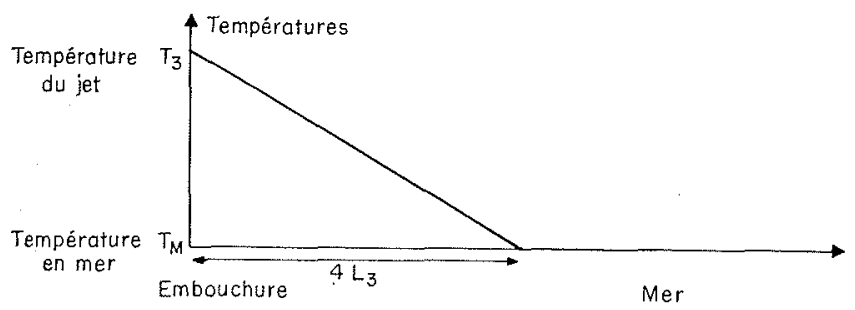

Si, d'une marée à l'autre, les eaux rentrant dans l'estuaire au flot ne sont pas du tout renouvelées, elles reviendront donc en suivant la loi de température ci-dessus, ce qui donne comme condition aux limites :

$$
T(t)=T_{3}-\left(\alpha / 4 L_{3}\right)\left(T_{3}-T_{M L}\right)
$$

avec :

$$
\alpha=\int_{0}^{t} \frac{|Q(t)|}{S(t)} d t
$$

(si $\alpha>4 L_{3} \quad, \quad T=T_{M}$ ).

$t=$ temps (origine au début du flot);

$S=$ section mouillée à l'embouchure;

$Q \quad=$ débit à l'embouchure;

On sera pessimiste en choisissant pour $T_{3}$ la valeur la plus forte des températures à l'embouchure au cours du jusant. On est pessimiste de toute façon, car les eaux sont renouvelées un minimum par l'effet d'aspiration qui intéresse une plus large étendue de mer que l'effet de jet au jusant.

Dans le cas où les courants marins renouvellent entièrement les volumes aspirés au flot, on imposera plutôt comme condition à l'embouchure la température $T_{\Delta L}$ de la mer.

\section{CONDITION A LA JONCTION ENTRE LES 3 BRAS :}

Cette jonction est assurée dans la partie hydraulique du modèle par le volume de confluent $\Omega$ occupé dans l'estuaire au droit de la prise. L'évolution de sa température sera représentée par

$$
\left(\partial \Omega T_{\omega} / \partial t\right)+Q_{1} T_{1}+Q_{2} T_{2}-Q_{3} T_{3}=0
$$

L'indice se rapporte à chacun des bras. $Q$ et $T$ représentent donc les débits et les températures à l'aval des bras 1 et 2 et à l'amont du bras 3 (le signe moins provient de l'orientation identique qui est donnée aux trois bras, de l'amont vers l'embouchure).

La thermique du confluent se présente donc ainsi :

- si $Q_{1}>0$, le bras 1 apporte au confluent de l'eau à la température $T_{1}$ :

$$
\frac{\partial T_{1}}{\partial t}+V_{1} \frac{\partial T_{1}}{\partial x}=0
$$

- si $Q_{1}<0$, le confluent apporte au bras 1 de l'eau à la température $T_{\omega}$ :

$$
\text { d'où } T_{1}=T \omega
$$

On impose des conditions analogues pour les bras 2 et 3 . Le test sur le signe de $Q_{3}$ est évidemment inversé.

\section{Application du modèle d'estuaire au site de Cordemais}

\section{Données de base des calculs}

\section{Données géométriques}

On trouvera, sur la figure 1 , la situation de Cordemais dans l'estuaire de la Loire. L'estuaire a été schématisé en un simple canal à section rectangulaire de largeur et de profondeur variables. On s'est surtout efforcé de respecter 


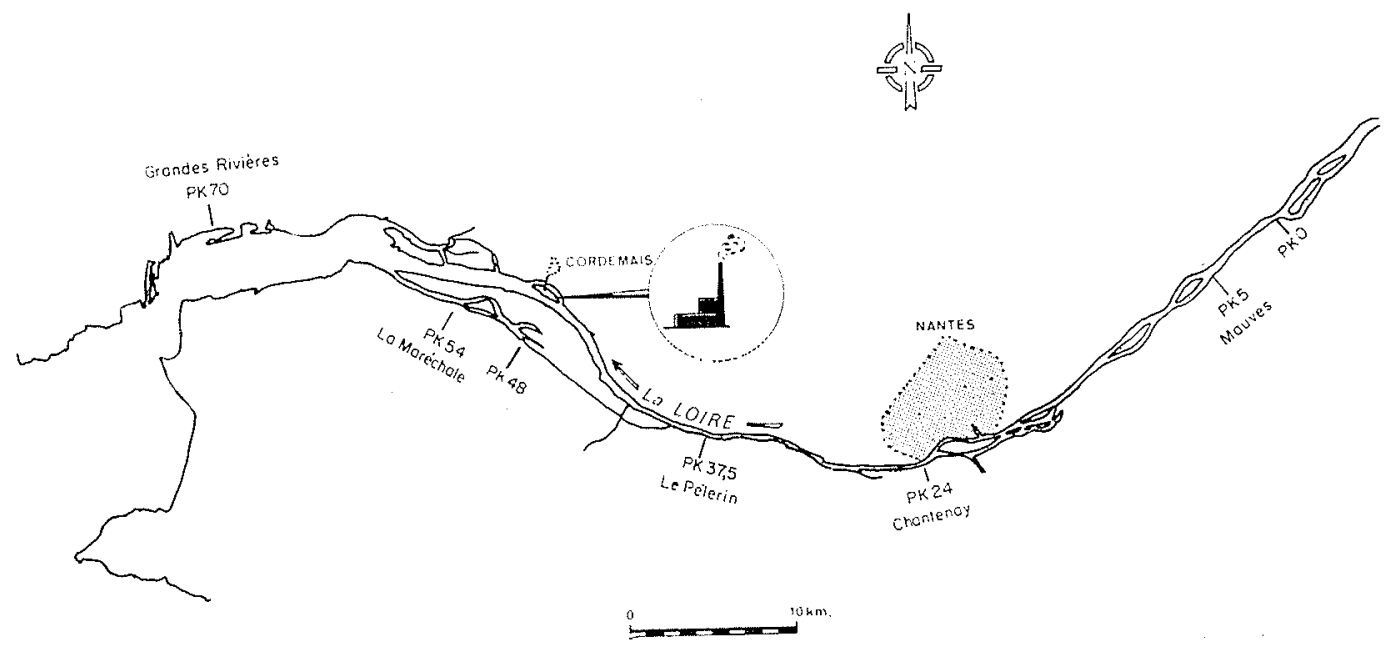

1/ Estuaire de la Loire. Situation de la centrale de Cordemais.

l'évasement de l'estuaire et la variation des sections avec la cote.

Sur la figure 1, le PK 48 indiqué pour Cordemais correspond à celui de l'extrémité du bras de restitution. Celuici constitue le bras 2 dans le modèle. Il est représenté par un canal long de $2 \mathrm{~km}$ et de $30 \mathrm{~m}$ de large. Le bras 1 va du PK 0 (soit un point situé à $5 \mathrm{~km}$ à l'amont de Mauves) jusqu'au confluent de PK 48. La prise est située au PK 46.

Le bras 3 va du confluent jusqu'à Grandes-Rivières (PK 70).

\section{Données hydrauliques}

Les calculs ont été effectués dans le cas où l'échauffement de l'estuaire, et donc la recirculation, sont maxima. On montre que ce cas est constitué par la conjonction de la période d'étiage en Loire avec une période de fortes marées. On a donc supposé la répétition de marées de coefficient 94 avec un débit en Loire de $150 \mathrm{~m}^{3} / \mathrm{s}$

La marée de coefficient 94 a été choisie en raison d'informations précises que l'on possédait à son sujet.

Le débit de prise est fixé par des considérations d'ordre thermique.

\section{Données thermiques}

Le problème de l'échauffement de lestuaire et de la recirculation directe ne se posant pas dans l'état actuel de façon aiguë, on a cherché à se placer d'emblée dans l'hypothèse d'une puissance rejetée d'environ $5800 \mathrm{MW}$. Cette puissance correspond à un débit de prise d'environ $175 \mathrm{~m}^{3} / \mathrm{s}$ pour un échauffement de $8{ }^{\circ} \mathrm{C}$ (en fait les eaux de prise sont échauffées de $12{ }^{\circ} \mathrm{C}$ mais on y ajoute un débit prélevé pour dilution juste à l'amont đu bras).

On aura pu noter que la partie thermique du modèle permet le calcul d'échauffements indépendamment des températures de base, celle du fleuve et celle de la mer (autrement dit ce sont des températures réduites qui interviennent). Mais le niveau même des températures atteintes peut avoir une importance en soi. Ce niveau présente des variations saisonnières liées aux échanges atmosphériques. Choisir des valeurs pour calculer les échanges atmosphériques sous-entend également le choix du niveau des températures. On s'est donc placé dans des conditions d'été : la température en Loire a été prise à $20^{\circ} \mathrm{C}$. La température en mer est également d'environ $20^{\circ} \mathrm{C}$ en été. Elle a été prise exactement à cette valeur si bien que, dans ces conditions, la température naturelle de l'estuaire est de $20^{\circ} \mathrm{C}$ partout.

Sur les indicaions de la Division Echanges Atmosphériques (E.D.F., Direction des Etudes et Recherches, Service G.E.C.T.E.N.) les échanges atmosphériques ont été calculés sur la base des valeurs suivantes (cf. plus haut pour la signification des paramètres):

$$
\begin{aligned}
\text { Teq } & =20{ }^{\circ} \mathrm{C} \\
a & =12 \mathrm{~W} / \mathrm{m}^{2}{ }^{\circ} \mathrm{C} \\
b & =7 \mathrm{~J} / \mathrm{m}^{3}{ }^{\circ} \mathrm{C} \\
u & =2 \mathrm{~m} / \mathrm{s} .
\end{aligned}
$$

\section{Qualité de la représentation hydraulique de l'estuaire}

On a vu que le modèle nécessite la connaissance des coefficients de frottement de Strickler. On s'est contenté d'adopter, pour ce coefficient $K$, des valeurs courantes sans s'attacher à un étalonnage coûteux $(K=60$ M.K.S. entre les $\mathrm{PK} 0$ et $15, \mathrm{~K}=40$ M.K.S. entre les $\mathrm{PK} 15$ et 70). En cela aussi l'estuaire est schématique.

La comparaison entre les courbes de marée mesurées et calculées dans différents PK de l'estuaire a montré une bonne concordance (les courbes mesurées ont été tirées du rapport cité en référence [1]). En ce qui ooncerne l'évolution des débits, la comparaison a été effectuée sur les courbes des cubes totaux introduits à l'amont des diverses sections. Elle s'est également avérée satisfaisante. On retrouve par ailleurs que Mauves (PK 5) est pratiquement à la limite maritime de l'estuaire. Au PK 0, le calcul donne une cote constante à $3 \mathrm{~cm}$ près.

On retiendra donc qu'une schématisation d'estuaire reproduit suffisamment bien le mécanisme de la convection 
pour le calcul des températures, les paramètres importants étant :

- les distances de parcours au flot et au jusant qui fixent le nombre de passages de mêmes eaux devant la centrale;

- les débits dans l'estuaire au droit du rejet et les débits de celui-ci qui fixent les échauffements successifs que les eaux subissent;

- le temps de transit total des eaux dans l'estuaire qui, avec la variation des cotes, règle l'influence des échanges atmosphériques;

- la variation des sections mouillées, qui, avec l'ordre de grandeur des débits, règle l'influence de la dispersion.

\section{Equilibre thermique de l'estuaire}

\section{Conditions de calcul}

La partie thermique du modèle nécessite la connaissance des courants en mer et de l'importance de la dispersion dont l'estuaire est le siège. A défaut d'étalonnage concernant ces deux points, on a effectué un calcul paramétrique comportant :

- pour le refroidissement par la mer, la prise en compte de deux cas, l'un optimiste et l'autre pessimiste. Dans le cas optimiste, on suppose que les eaux pénétrant au flot sont entièrement froides $\left(20^{\circ} \mathrm{C}\right)$. Dans le cas pessimiste, on suppose que ce sont les masses évacuées au jusant qui reviennent au flot dans les conditions exposées plus haut,

- pour le coefficient de dispersion, la prise en compte de valeurs différentes de $\alpha$ et $\beta$ dans la loi qui le régit (cf. $\$ 2$ ). On a d'abord retenu les valeurs obtenues par Prichard [3] pour l'estuaire de l'Hudson, soit :

$$
D_{x}=65(Q / L)+10
$$

Cette formule conduit à des valeurs de $D_{x}$ variant entre 0 et $250 \mathrm{~m}^{2} / \mathrm{s}$ suivant l'état de la marée (pour la marée de coefficient 94 et le débit en Loire de $150 \mathrm{~m}^{3} / \mathrm{s}$ ). Ces valeurs sont pessimistes en regard de celles observées par Fischer [7] dans l'estuaire de la Mersey $\left(500 \mathrm{~m}^{3 / \mathrm{s}}\right)$. Elles sont, en tout cas, d'un ordre de grandeur courant et nous les dirons vraisemblables (mais déjà pessimistes) par rapport à la seconde série de valeurs retenues qui correspondent aux premières divisées par 2. D'où :

$$
D_{x}=32,5(Q / L)+5
$$

L'adoption de deux lois pour le refroidissement par la mer et de deux lois pour la dispersion conduit donc à trois cas de calculs qui comportent :

Cas $A$ :

- Refroidissement par la mer pessimiste :

$$
T=T_{3}-\left(\alpha / 4 L_{3}\right)\left(T_{3}-20\right)
$$

(cf. partie thermique du modèle)

- Dispersion yraisemblable $: D_{x}=65(Q / L)+10 \mathrm{~m}^{2} / \mathrm{s}$
Cas B :

- Refroidisement par la mer optimiste : $T=20^{\circ} \mathrm{C}$

- Dispersion vraisemblable : $D_{x}=65(Q / L)+10 \mathrm{~m}^{2} / \mathrm{s}$

Cas C :

- Refroidissement par la mer pessimiste :

$$
T=T_{3}-\left(\alpha / 4 L_{3}\right)\left(T_{3}-20\right)
$$

(cf. partie thermique du modèle)

- Dispersion pessimiste $: D_{x}=32,5(Q / L)+52 / \mathrm{s}$.

Le cas A sera le cas de référence.

\section{Echauffement de l'estuaire}

La comparaison des cas $A$ et $B$ donne l'influence du refroidissement par la mer, celle des cas $\mathrm{A}$ et $\mathrm{C}$ donnant linfluence de la dispersion.

On pourra effectuer ces comparaisons au vu des fig. 2 et 3 . On y a porté les évolutions de la courbe des températures le long de l'estuaire au cours d'une marée, une fois le régime d'équilibre atteint (dans les calculs, l'état initial correspond à l'état naturel). On reconnaîtra, sur les figures, les mouvements de flot et de jusant au sens de déplacement des points de température maximum.

La comparaison des courbes montre que l'écart obtenu dans les trois cas est faible et que le paramétrage effectué encadre le résulat réel de façon assez fine : la prise en compte d'hypothèses moins grossières pour le refroidissement par la mer, et l'étalonnage même approximatif de la dispersion dans l'estuaire, rendront le modèle très représentatif.

Il faut, par ailleurs, vingt marées pour que le régime d'équilibre soit atteint à partir d'un profil des températures plat. Il est bien évident que, dans la nature, on ne trouvera jamais une succession de marées fortes dépassant le nombre de 5 ou 6. Mais elles n'agiront pas à partir d'un profil des températures plat et c'est la raison pour laquelle on peut avancer les résultats suivants :

- pour une puissance rejetée à Cordemais de $5800 \mathrm{MW}$, il faudra s'attendre, en période de fortes marées et débit d'étiage, à un échauffement pouvant atteindre 6 à $7{ }^{\circ} \mathrm{C}$ entre Cordemais et Paimbouf (PK 60); la température décroît ensuite linéairement de Paimbœuf à l'embouchure;

- l'influence de la centrale se fera sentir jusqu'à Chantenay (PK 24) à certaines heures de la marée. D'où le risque d'interférences avec la centrale de Cheviré (PK 27).

\section{La recirculation}

En ce qui concerne la recirculation, la figure 4 donne dans les trois cas l'évolution des températures moyennes :

— du rejet, c'est-à-dire à l'extrémité du bras;

- du mélange qui en résulte au confluent;

- dans la section au droit de la prise.

On remarquera que la température de prise est toujours supérieure à $20^{\circ} \mathrm{C}$, c'est-à-dire qu'il y aura une recircu- 

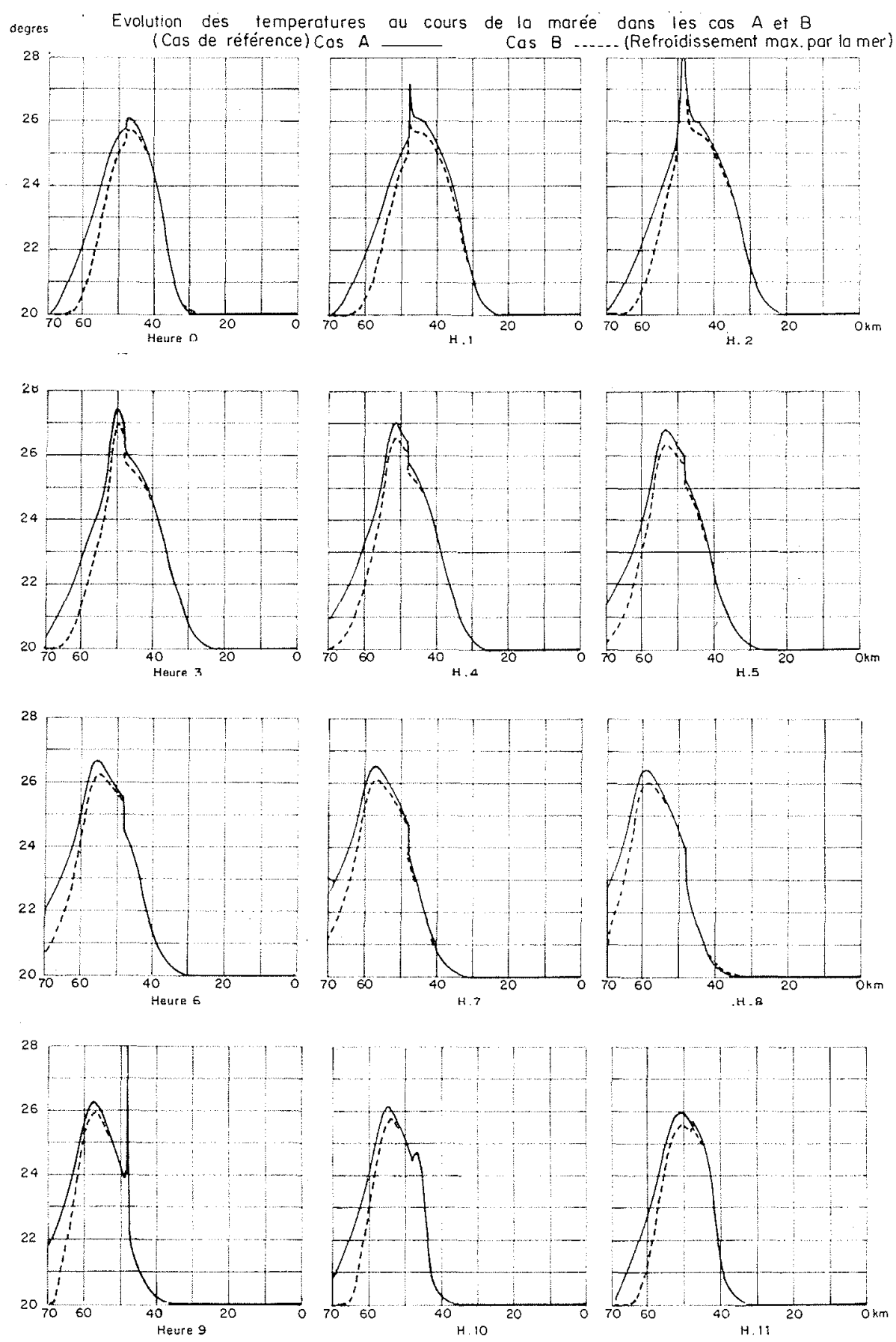

2/ Influence du refroldissement par la mer. 

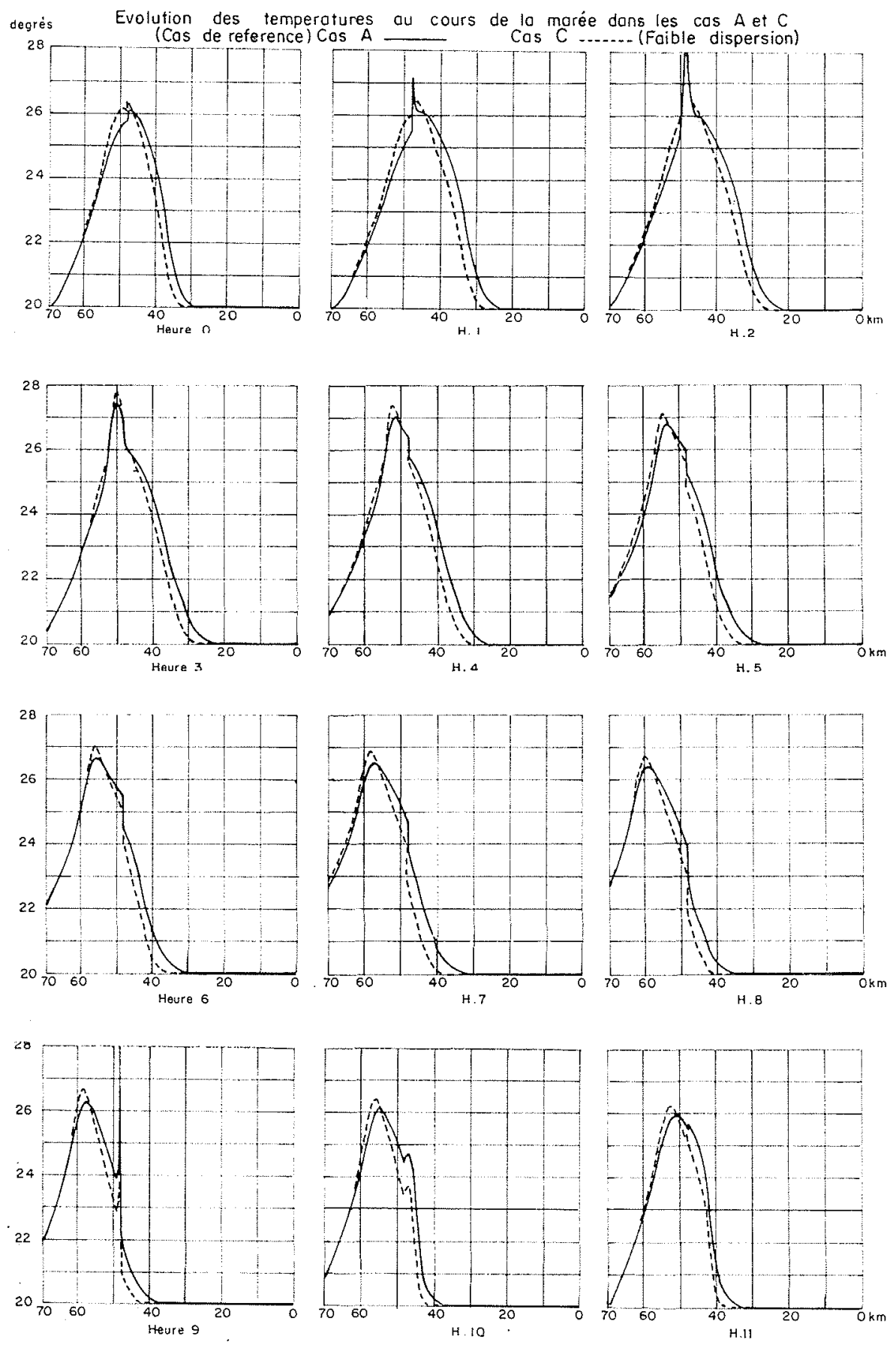

3/ Influence de la dispersion. 
lation différée permanente. Son minimum est atteint à la fin du jusant. Il concerne donc, parmi les eaux pompées par la centrale au cours de la marée, celles qui étaient situées le plus à lamont à la marée précédente et qui n'étaient encore jamais passées devant la prise, étant donné la dérive imposée par le débit fluvial.

C'est un effet de la dispersion et l'on voit l'intérêt qu'il y aura à la préciser. Puisqu'il s'agit d'un effet de la dispersion, on tient là le minimum de la recirculation différée auquel il faudra s'attendre. Si on élimine le cas C, pour lequel la dispersion est trop faible, on voit sur la figure 4 que ce minimum est de $1,5^{\circ} \mathrm{C}$.

La recirculation directe sera maximum au moment de la renverse du jusant vers le flot pour deux raisons :

- le bras rejette des eaux chaudes en milieu pratiquement calme, d'où très peu de dilution longitudinale, contrairement aux cas où il y a de forts débits;

- l'accélération due au flot fait ensuite remonter les eaux chaudes jusqu'à la prise avant qu'elles aient eu le temps de se disperser latéralement; elles restent donc concentrées du côté de la berge de la prise, d'où une recirculation directe maximum.

Parmi toutes les tranches qui passent devant la prise, celles qui s'étaient trouvées devant le rejet, au moment de la renverse de jusant à flot, seront donc caractérisées par des calories :

- en nombre maximum;

- concentrées du côté de la prise.

Cet effet de recirculation directe est traduit qualitativement, sur la figure 4, par le pic de température qui se forme au droit du rejet au moment de la renverse de jusant à flot (courbes $T_{\Omega}$; on aura pu également observer ce pic sur les figures 2 et 3 ). Le pic remonte ensuite vers la prise en s'atténuant rapidement sous l'effet de la dispersion longitudinale des calories, de section droite à section droite (on peut d'ailleurs constater cet effet sur les figures 2 et 3 ). L'effet de pic se produit sur les tranches d'eau venant de l'amont et de température déjà portée à $22,5^{\circ} \mathrm{C}$ sous l'effet de la recirculation différée (dans les cas $\mathrm{A}$ et $\mathrm{B}$ résultat non porté sur les courbes). Ces tranches reçoivent une eau rejetée par le bras à environ $30^{\circ} \mathrm{C}$ (cf. fig. 4, température $T_{r}$ ). Elles repartent ensuite vers l'amont et arrivent devant la prise $1 \mathrm{~h} 10$ après leur renverse. On voit, sur la figure 4 , que leur température atteint alors $24,5{ }^{\circ} \mathrm{C}$ (éliminons le cas $\mathrm{C}$ pour lequel la dispersion est trop faible). Mais ceci est une température minimum pour la prise, correspondant à un mélange latéral total. En fait, les eaux rejetées à $30^{\circ} \mathrm{C}$ restent concentrées sur la berge de la prise (la température vers l'autre berge étant de $22,5^{\circ} \mathrm{C}$ ).

On aura donc une température des eaux prélevées comprise entre $24,5^{\circ} \mathrm{C}$ et $30^{\circ} \mathrm{C}$, d'où une recirculation directe comprise entre :

$$
\begin{aligned}
30-22,5 & =7,5{ }^{\circ} \mathrm{C} \\
\text { et } 24,5-22,5 & =2{ }^{\circ} \mathrm{C}
\end{aligned}
$$

qui s'ajoutera aux $2,5^{\circ} \mathrm{C}$ de recirculation différée.

\section{Conditions d'évacuation de l'énergie apportée par la centrale}

Si le paramétrage effectué joue peu sur le niveau des températures, il n'en va pas de même pour les énergies. Ceci tient à l'importance des masses intéressées par les températures. L'évacuation des $70 \mathrm{GWh}$, apportées à chaque marée par la centrale, se fait de la manière suivante :

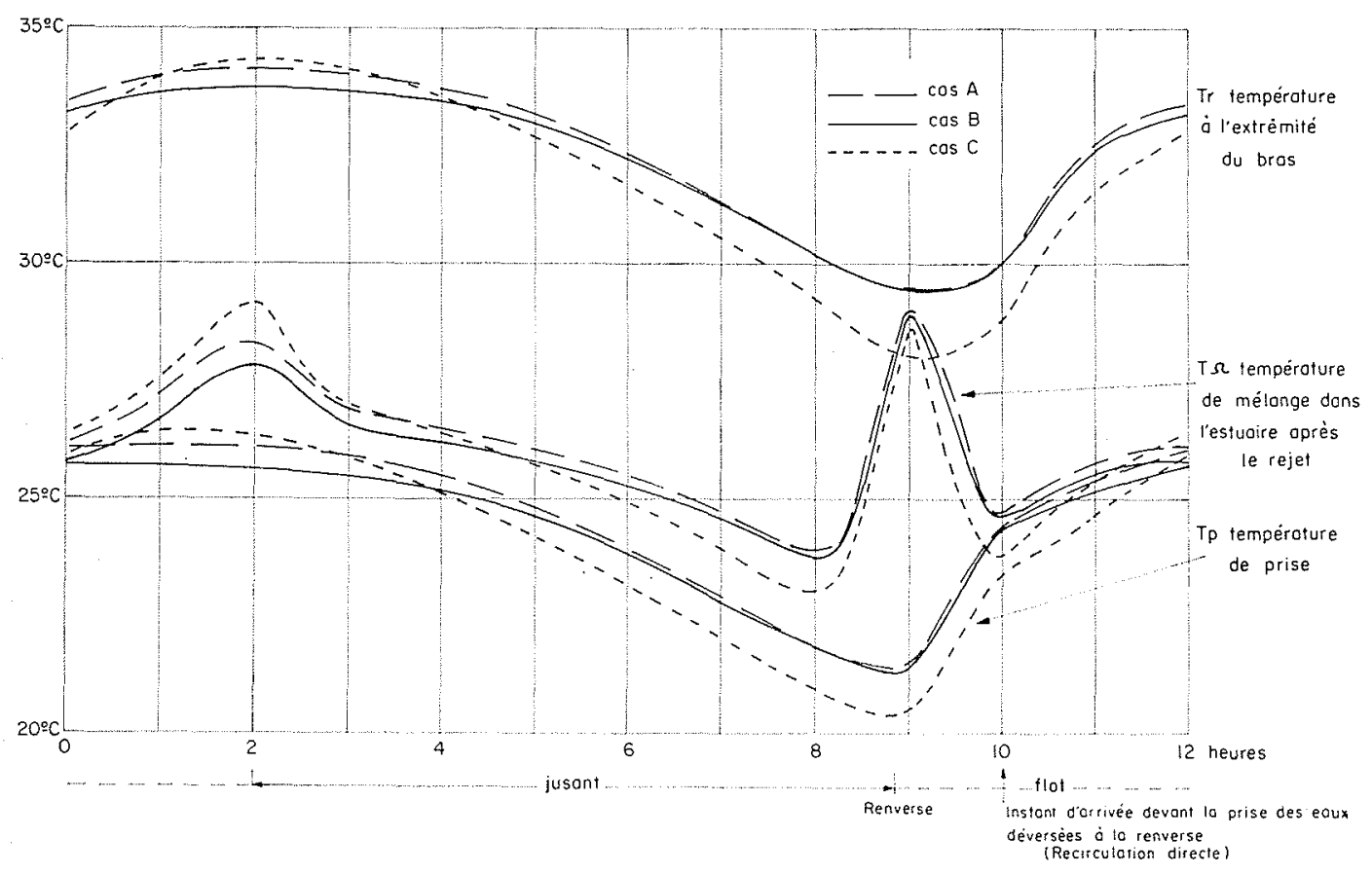

4/ Evolution des températures au droit de Cordemais. 


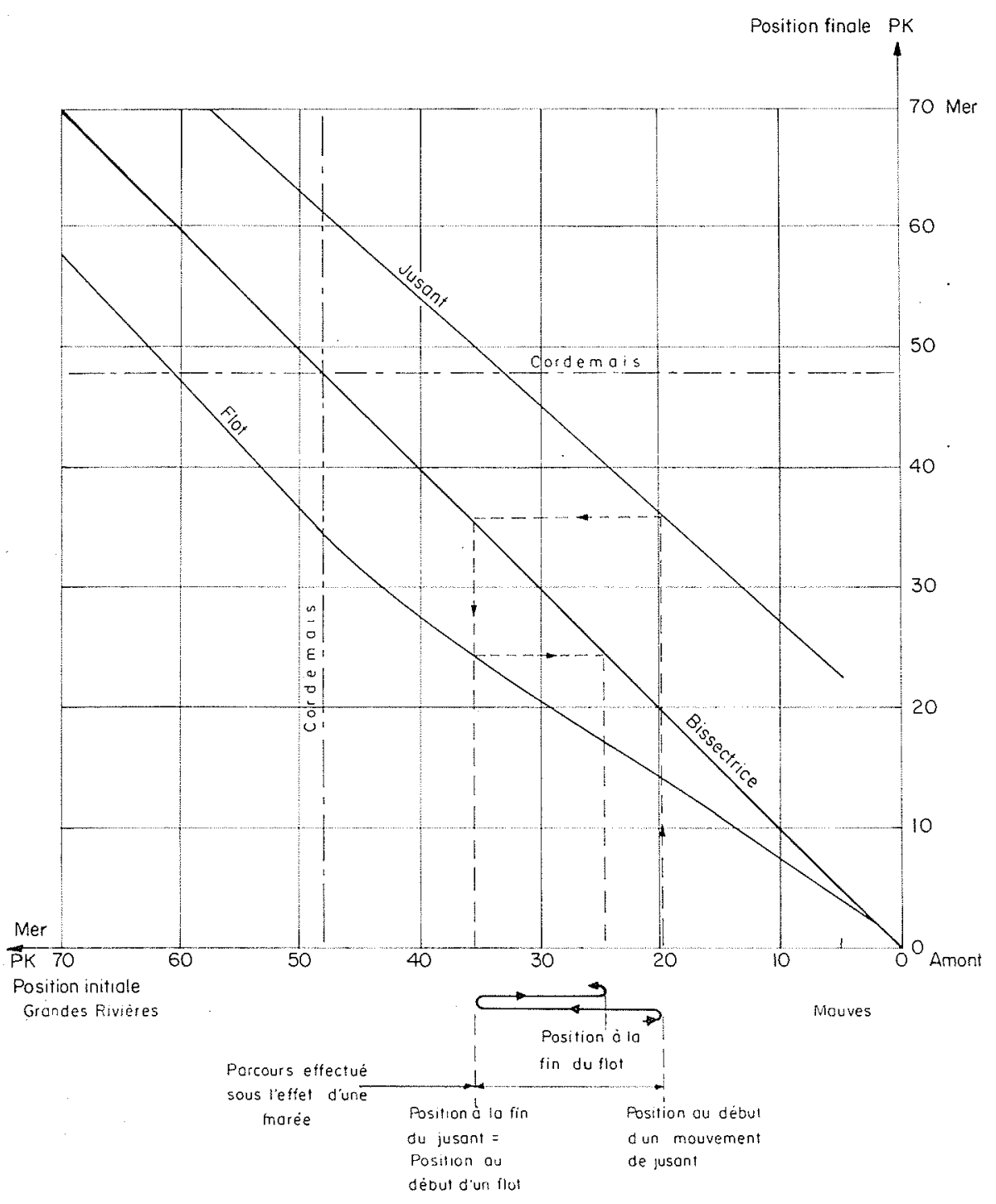

En abscisse: position d'une tranche d'eau dans l'esiuaire à l'instant où elle subit une renverse soit vers un mouvement de

En ordonnée: position à la fin, soit du flot, soif du jusant.

En pointillé: exemple de parcours subi par une tranche.

5/ Distances parcourues au flot et au jusant

\begin{tabular}{|c|c|c|c|}
\hline CAS & $\begin{array}{c}\text { A } \\
\text { Cas } \\
\text { de } \\
\text { jéférence }\end{array}$ & $\begin{array}{c}\text { B } \\
\text { Fort } \\
\text { refroidis- } \\
\text { sement } \\
\text { parla mer }\end{array}$ & $\begin{array}{c}\mathrm{C} \\
\begin{array}{c}\text { Faible } \\
\text { dispersion }\end{array}\end{array}$ \\
\hline $\begin{array}{c}\text { Energie évacuée par la } \\
\text { mer } \ldots \ldots \ldots \text { (GWh) }\end{array}$ & 18 & 55 & 13 \\
\hline $\begin{array}{c}\text { Energie évacuée par l'at- } \\
\text { mosphère .... (GWh) }\end{array}$ & 50 & 14 & 48 \\
\hline $\begin{array}{r}\text { Energie contenue dans l'es- } \\
\text { tuaire à l'heure } 0 .(\mathrm{GWh})\end{array}$ & 920 & 580 & 904 \\
\hline
\end{tabular}

On peut remarquer que, pour le cas $C$, le calcul a été arrêté avant que l'équilibre exact ait été atteint (le total des énergies évacuées n'atteint pas $70 \mathrm{GWh}$ ). L'expérience des calculs $A$ et $B$ avait montré que la différence qui en résulte, entre les températures d'équilibre réelles et les températures obtenues à ce stade du calcul, est inférieur au dixième de degré. Or, on s'est surtout attaché à obtenir les températures. Mais si l'on désire s'attacher au mode d'évacuation de l'énergie déversée, le tableau montre qu'il est nécessaire de préciser les paramètres. On voit que l'action de la mer peut inverser complètement le rapport des deux postes de refroidissement : celui qu'elle représente et celui de l'atmosphère. Un bon refroidissement par la mer offre corrélativement l'avantage de maintenir en permanence moins de calories dans l'estuaire.

La comparaison des cas $\mathrm{A}$ et $\mathrm{C}$ montre l'influence de la dispersion sur le refroidissement par la mer. Si lon fait une règle de trois sur les chiffres obtenus dans le cas $\mathrm{C}$, on trouve :

\énergie évacuée par la mer ......... 15 énergie évacuée par l'atmosphère ....... 55 énergie contenue dans l'estuaire ...... 1030

On trouve donc ici que diviser le coefficient de dispersion par 2, fait décroître le refroidissement par la mer d'environ $15 \%$ et accroît le refroidissement de l'atmosphère d'environ $10 \%$. 


\section{Conclusions}

\section{Résultats atteints}

On pourrait songer à mettre, à la disposition du projeteur, un moyen simplifié d'évaluer les échauffements dans les estuaires, à la lumière des enseignements tirés des premières exploitations du modèle.

Ceci peut effectivement se faire en ce qui concerne la partie thermique, en jouant sur les échelles de temps des trois modes d'échanges : la convection, les échanges atmosphériques et la dispersion (dans l'ordre croissant pour les échelles de temps). Malheureusement, il est alors nécessaire de connaître le nombre des passages de mêmes eaux devant la centrale et cette connaissance passe, obligatoirement, par l'utilisation du calcul hydraulique (la fig. 5, tirée des résultats du calcul, montre comment évaluer le nombre de passages dans le cas de marées de coefficient 94 répétées; il est de 18). En effet, raisonner sur des vitesses moyennes pendant le jusant et le flot conduirait à un nombre totalement erroné de 7 à 8 passages.

La non-linéarité des lois qui régissent la mécanique de l'estuaire ne peut donc pas être négligée. Puisqu'il est nécessaire d'utiliser le modèle au moins dans sa première partia (la partie hydraulique), il n'est pas gênant d'utiliser aussi la seconde (la partie thermique), qui représente un volume de calculs relativement faible dans le volume total.

Par ailleurs, on aura remarqué l'importance du rôle de la dispersion sur les effets de pic de température aux renverses de courant, ainsi que son influence sur le refroidissement par la mer.

Ces conclusions constituent, en quelque sorte, des premiers résultats. En ce qui concerne les résultats proprement dits de l'exploitation du modèle pour l'estuaire de la Loire, ils peuvent se résumer ainsi :

Une première exploitation du modèle a été faite pour estimer l'échauffement maximum qu'une puissance rejetée de $5800 \mathrm{MW}$ installée sur le site de Cordemais pourra apporter dans l'estuaire de la Loire. Cet échauffement, à l'aval de la centrale, est de l'ordre de 6 à $7^{\circ} \mathrm{C}$ par fortes marées et en période d'étiage en Loire. La surélévation de la température naturelle, au niveau de la prise, atteindra en moyenne $4{ }^{\circ} \mathrm{C}$ (minimum $1,5^{\circ} \mathrm{C}$ et maximum $6{ }^{\circ} \mathrm{C}$ ). Au moment de la renverse des courants vers le flot, cette recirculation différée sera de $2,5^{\circ} \mathrm{C}$. Il s'y ajoutera une recirculation directe comprise entre 2 et $7,5^{\circ} \mathrm{C}$.

Ces résultats ont été présentés pour une puissance rejetée déterminée afin de fixer d'emblée les idées sur les ordres de grandeurs mis en jeu. On peut cependant extrapoler, à d'autres valeurs de la puissance rejetée, par simple règle de trois. Ceci provient de la linéarité de l'équation de la température (on considère par ailleurs que le champ des vitesses dans l'estuaire ne sera pas modifié de façon significative si l'on fait varier les débits de refroidissement de la centrale).

\section{Performances atfendues}

Le modèle, tel qu'il est actuellement, peut donc être utilisé pour préciser la puissance implantable. Il sera cependant amélioré par l'étalonnage de la dispersion et de l'action de la mer afin d'éviter un paramétrage coûteux. L'étalonnage peut se faire à partir de l'évolution de la salinité dans l'estuaire. Par contre, si l'on veut utiliser le modèle comme instrument d'optimisation, soit de l'implantation de plusieurs centrales le long de l'estuaire, soit du dimensionnement du bras pour favoriser le stockage (ce qui limite dans le temps la recirculation directe), ni l'étalonnage ni le paramétrage ne sont nécessaires car il s'agit de problèmes purement convectifs.

Une extension du modèle sera par ailleurs entreprise afin de pouvoir répondre quantitativement aux problèmes posés par la recirculation. Pour cela, une description plus fine des mécanismes locaux entre prise et rejet sera introduite. Cette description pourra d'ailleurs tenir simplement dans la séparation «en deux fils» du modèle filaire actuel. Des modèles, tels que ceux cités en référence [4] et [5], prennent ainsi en compte la disparité entre la température du jet remontant au flot vers la prise et la température du débit de l'estuaire. Bien entendu, les deux fils communiquent.

Enfin, il faut remarquer que la notion de puissance implantable n'est pas uniquement attachée aux bornes des températures atteintes. Elle est aussi liée à leur fréquence d'apparition. Pour lobtenir, il est nécessaire de reproduire, en tout ou partie, la succession des coefficients de marée des débits fluviaux et des saisons, éléments déterminants du régime thermique de l'estuaire. L'étude doit être poursuivie dans le sens d'une réduction des temps de calculs, soit en s'affranchissant de l'échelle des temps de la marée par un résumé de son action, soit en se limitant à l'étude d'une suite discrète d'occurrences des trois paramètres cités. Il est dès maintenant possible de mettre en cuvre le dernier de ces moyens, bien qu'il représente encore un certain volume de calcul.

\section{Références}

[1] «Etude sur modèle réduit de la Loire Atlantique », Laboratoire National d'Hydraulique, série A (octobre 1958)

[2] JACOUET ( $J$.) et GRAS (R.). - Les échanges d'énergie entre un plan d'ean et l'atmosphère : détermination de la capacité de réfrigération d'un plan d'eau. $X I^{\circ}$ Joumées de l'Hydraulique (S.H.F.), question IV, rapport 12 .

[3] Prichard (D.W.), Akira Okubo et Mehr (E.). - A study of the movement and diffusion on an introduced-contaminant in New-York Harbor Waters. Chesapeake Bay Institute, Technical Report 31, p. 28, ref. 62-61 (october 1952).

[4] Stefan (H.) et Vaidyaraman (P.). - Jet type model for the three Dimensional thermal plume in a crosscurrent and under wind. Water Resources Research, vol. 8, number 4, p. 998 (août 1972).

[5] Stolzenbach (K.D.) et Harleman (D.R.F.). - An analytical and experimental investigation of surface discharges of heated water. M.I.T., Department of Civil Engineering, Report no 135 (févriè 1971).

[6] Fischer (H.B.). - Transport mechanisms in partially stratified estuaries. Jotrnal of Fluid Mechanics, vol. 53, part 4, p. 671 (1972).

[7] Schilchting (M.). - "Grensschichtheorie ». Verlag G. Braun, Karlsruhe, chapitre 24, p. 673 .

Voir la Discussion à la page suivante 


\section{Discussion}

Président : M. M. Hug

M. le Président remercie M. Malherbe pour son exposé consacré à un sujet d'actualité. Il ouvre ensuite la discussion.

M. Toureau (E.D.F., Equipement) pose les deux questions ciaprès :

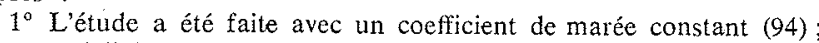
or, en réalité, ce coefficient varie d'une marée à l'autre. A-t-on une idée de l'influence de cette variation sur la dispersion des calories dans l'estuaire et vers la mer?

Il semble que les marées de mortes eaux devraient être favorables à cette dispersion.

$2^{\circ}$ En principe, les matières en suspension dans l'eau de l'estuaire modifient les coefficients d'échange eau-atmosphère et les coefficients de correction. A-t-on une idée de l'importance de cette influence?

C'est en première approximation et pour alléger les calculs répond M. Malherbe, que l'on a admis un coefficient de marée constant, mais il est probable que les faibles valeurs de ce coefficient devraient atténuer la valeur des températures maximales dans l'estuaire.

En ce qui concerne l'influence de la turbidité sur les échanges de chaleur, M. Malherbe se propose de consulter les spécialistes qui lui ont fourni les éléments nécessaires à la description de ces échanges dans la région de la Loire.

M. Gras (E.D.F., Equipement) s'intéresse aux échanges de masse et de chaleur à l'extrémité aval de l'estuaire, c'est-à-dire avec la mer. Il semble que ces échanges - résultant du fiux et du reflux puissent être importants et avoir une grande influence sur la quantité d'énergie stockée dans l'estuaire.

D'autre part, il pense que le modèle présenté donne des résultats un peu pessimistes en ce qui concerne les échanges atmosphériques du fait :

$1^{\circ}$ que l'on a adopté des coeflicients de transferts relativement faibles;

$2^{\circ}$ que l'on n'a pas tenu compte de la stratification thermique (verticale et horizontale) que l'on observe souvent dans la nature et qui fait que l'eau la plus chaude se trouve généralement à la surface.

Sur le premier point, M. MaLHerbe répond que le modèle montre que les échanges de masse et de chaleur, à l'aval de l'estuaire, modifient peu les températutres maximales à l'intérieur de ce dernier, malgré les importantes quantités de chaleur qu'ils peuvent éventuellement représenter. Cela dans le cas considéré où la centrale thermique est implantée à l'amont de l'estuaire, observe
M. Toureau. Cela tient, dit M. Malmerbe à l'importance des masses d'eau intéressées près de l'embouchure, très large. Un faible écart de température représente, pour ces masses, des différences d'énergie considérables.

Sur le deuxième point, un débat s'instaure entre M. Malmerbe, M. Berthois, M. Gras et M. Maquet.

M. MALherbe fait remarquer que la nappe d'eau chaude que l'on observe en nature correspond à l'eau qui vient d'être rejetée par la centrale. Cette eau se disperse ensuite en profondeur et conduit à un échauffement dans la masse de l'estuaire. C'est cet échauffement dans la masse que les calculs représentent. ll s'agit là d'un effet à long terme. Une même tranche d'eau va jusqu'à passer 18 fois devant la centrale de Cordemais, alors que les panaches d'eau chaude dont il est question se dispersent en un nombre de marées de l'ordre de l'unité dans les estuaires dits mélangés. L'estuaire de la Loire est à ranger dans cette catégorie comme l'ont montré les mesures effectuées par le Laboratoire National d'Hydraulique.

En réponse à l'une des questions posées, $M$. Berthors signale les deux publications suivantes relatant les observations faites en nature sur les déplacements d'une tranche d'eau sous l'effet de la marée et du débit fluvial :

1. L. Berithors. - Observation d'une tranche d'eau suivie dans ses déplacements entre Basse-Indre et Saint-Nazaire. Cahiers Océanographiques. Bull. C.O.E.C., tome X., fasc. 4, avril 1958, p. $190-208$.

2. L. Berthors et Mlle Y. Le Gullly. - La stratification des eaux dans l'estuaire de la Loire. C.R. Ac. Sc. Paris, séance du 6 mai 1963 , t. 256 , p. $4060-4063$.

M. le Président souligne l'intérêt des divers points qu viennent d'être débattus; en particulier, la validité de l'hypothèse suivan laquelle la Loire présente un estuaire bien mélangé est à contrôler : certaines mesures exécutées à Cordemais semblent montrer que la nappe d'eau chaude se trouve en surface, ce qui confirme certains résultats donnés par $M$. le Professeur HaRLeman.

M. le Président suggère à $M$. Malherbe de prendre des informations complémentaires en vue de pouvoir répondre avec le maximum de clarté - dans le procès-verbal de la discussion - aux intéressantes questions qui lui ont été posées.

Il clôt ensuite la discussion en remerciant toutes les personnes qui y ont participé et donne la parole à M. DAubert pour l'exposé de sa communication. 